\title{
Throughput Fairness in Cognitive Backscatter Networks With Residual Hardware Impairments and a Nonlinear EH Model
}

\section{Xiaona Gao}

Xi'an University of Posts and Telecommunications https://orcid.org/0000-0001-8830-9464

Liqin Shi ( $\square$ liqinshi@hotmail.com )

Xi'an University of Posts and Telecommunications https://orcid.org/0000-0002-1200-8160

Guangyue Lu

Xi'an University of Posts and Telecommunications

\section{Research Article}

Keywords: Cognitive backscatter network, Energy harvesting, Hardware impairments, Fairness, Convex optimization

Posted Date: November 9th, 2021

DOI: https://doi.org/10.21203/rs.3.rs-1031589/v1

License: (c) (i) This work is licensed under a Creative Commons Attribution 4.0 International License. Read Full License

Version of Record: A version of this preprint was published at EURASIP Journal on Wireless Communications and Networking on February 8th, 2022. See the published version at https://doi.org/10.1186/s13638-022-02093-8. 


\title{
RESEARCH
}

\section{Throughput fairness in cognitive backscatter networks with residual hardware impairments and a nonlinear $\mathrm{EH}$ model}

\author{
Xiaona Gao, Liqin Shi* and Guangyue Lu
}

${ }^{*}$ Correspondence:
liqinshi@hotmail.com
Shaanxi Key Laboratory of
Information Communication
Network and Security, Xi'an
University of Posts \&
Telecommunications, Xi'an
710121, China
Full list of author information is
available at the end of the article

\begin{abstract}
This paper is to design a throughput fairness-aware resource allocation scheme for a cognitive backscatter network (CBN), where multiple backscatter devices (BDs) take turns to modulate information on the primary signals and backscatter the modulated signals to a cooperative receiver $(C-R x)$, while harvesting energy to sustain their operations. The nonlinear energy harvesting $(\mathrm{EH})$ circuits at the BDs and the residual hardware impairments (HWIs) at the transceivers are considered to better reflect the properties of the practical energy harvesters and transceiver$s$, respectively. To ensure the throughput fairness among BDs, we formulate an optimization problem to maximize the minimum throughput of BDs by jointly optimizing the transmit power of the primary transmitter, the backscattering time and reflection coefficient for each $\mathrm{BD}$, subject to the primary user's quality of service (QoS) and BDs' energy-causality constraints. We introduce the variable slack and decoupling methods to transform the formulated non-convex problem, and propose an iterative algorithm based on block coordinate descent (BCD) technique to solve the transformation problem. We also investigate a special CBN with a single BD and derive the optimal solution in the closed form to maximize the BD's throughput. Numerical results validate the quick convergence of the proposed iterative algorithm and that the proposed scheme ensures much fairness than the existing schemes.
\end{abstract}

Keywords: Cognitive backscatter network; Energy harvesting; Hardware impairments; Fairness; Convex optimization

\section{Introduction}

Internet of Things (IoT) is a breakthrough application for wireless communication systems. The ultimate goal of IoT is to make everything (human being, machine and things) achieve ubiquitous connectivity, which requires a large number of spectrum resources [1]. Cognitive radio (CR) enables the IoT nodes to share spectrum with primary users while ensuring that the interference from the IoT nodes does not exceed a predefined threshold, and thus improves spectral efficiency [2]. However, in conventional CR networks, the IoT nodes realize information transmissions by active radios that require power-hungry radio frequency $(\mathrm{RF})$ components, such as mixers, oscillators and analog-digital converters (ADC), leading to a high power consumption and greatly reducing the operation time of IoT nodes [3]. This issue poses another key challenge for the deployment of massive energy-constrained IoT devices. 
Recently, backscatter communication has gained tremendous momentum due to its ability of realizing extra-low-power or even battery-free information transmission. The key idea of backscatter communications is to allow IoT devices, also called backscatter devices (BDs) in this paper, to modulate their information bits over the incident RF signals by adjusting the antenna impedances and to reflect the modulated signals to the associated receivers, while harvesting energy to support their circuit operations [4]. Backscatter communications can be classified into three types, i.e., monostatic backscatter communication system, bistatic backscatter communication system, and ambient backscatter communication (AmBC) system. The monostatic backscatter communication system and bistatic backscatter communication system require dedicated RF sources to generate carrier wave signals, leading to an extra cost. On the contrary, the AmBC system leverages the existing RF signals (e.g., WiFi, Cellular signals), which eliminates the need for the deployment of dedicated RF sources and enjoys a low cost. Meanwhile, we note that in $\mathrm{CR}$, the primary signals can be the existing RF signals required by AmBC. This offers an opportunity to integrate AmBC into CR seamlessly and yields a cognitive backscatter network (CBN) for IoT [5].

The authors of [6] considered a CBN with single user, and maximized the ergodic capacity of a BD by jointly optimizing the primary user's transmit power and the BD's reflection coefficient. In [7], the authors extended the single user scenario in [6] to the multi-user $\mathrm{CBN}$ where non-orthogonal multiple access (NOMA) technology is applied to improve the spectral efficiency, and jointly optimized primary user's transmit power and BDs' reflection coefficients to maximize the sum-rate of BDs. In [8], the hybrid backscatter-active transmission was considered and a resource allocation scheme was developed to maximize the overall throughput of IoT devices by balancing the time for backscatter transmission and active transmission. In [9], a joint design for time scheduling, reflection coefficient of BDs together with the transmit power allocation of primary user was proposed in full-duplex-enabled CBN to maximize the throughput of backscatter system, while guaranteeing the minimum rate requirements of the primary system. The authors of [10] maximized the transmission rate of a BD by jointly optimizing the time and power allocation, subject to the minimum rate requirement of the primary system. In [11], the authors investigated the optimal tradeoff between the energy harvesting and backscatter modes, and the optimal reflection coefficient of BD to maximize the throughput of IoT node. Additionally, in [12], the throughput maximization problems for BDs were studied under the premise of the primary user's throughput requirement and BDs' energy-casuality constraints in single- and multi-user CBNs, respectively.

We note that the works mentioned above [6]-[12] have two limitations. On the one hand, these works assumed an ideal hardware for all active transceivers. However in practice, the hardware impairments (HWIs) exist in RF front ends of active transceivers due to the non-ideal characteristics of electronic circuit, which includes the nonlinearity of high-power-amplifier (HPA) and the phase noise caused by oscillators $[13,14]$. On the other hand, the linear power conversion efficiency was considered, which does not agree with the fact that the property of practical energy harvesting $(\mathrm{EH})$ circuits is non-linear $[15,16]$. Due to the above ideal assumptions, the existing resource allocation problems do not match the practical scenarios well 
and this may lead to resource allocation mismatches. Accordingly, both the problem formulation and its solution need to be revisited.

Inspired by this, in this paper, we formulate a max-min throughput problem for a CBN with multiples BDs while considering the HWIs and a non-linear energy harvesting model, and propose an iterative algorithm to solve it. Our main contributions are listed as follows:

(1) A max-min throughput problem is formulated to ensure fairness among all BDs. To be specific, the minimum throughput of BDs is maximized by jointly optimizing the transmit power of primary transmitter, and the backscattering time and reflection coefficient of each $\mathrm{BD}$, subject to the quality of service (QoS) constraint of primary user and energy-causality constraints of BDs. Please note that in our formulated problem, the practical nonlinear EH model at BDs and HWIs at active transceivers are taken into account, which increases the complexity at optimization stage since the EH function is fractional and the existence of HWIs introduce an additional component to the logarithm objective function with fractional structure. The formulated problem happens to be non-convex, and thus, there is no systematic or computationally-efficient approach to solve it directly.

(2) To solve the non-convex problem, we adopt the following three steps. First, we introduce a slack variable to transform the objective function into a linear one. Second, we determine the optimal transmit power to decouple it from the optimization problem. Third, we propose an iterative algorithm based on block coordinate descent (BCD), and obtain the sub-optimal solutions for the backscattering time and reflection coefficient of each BD. Besides, we study the special scenario only with a single $\mathrm{BD}$, and derive the closed-form expressions for the optimal time and reflection coefficient.

(3) Simulation results validate the following two results. First, the convergence value of the proposed iterative algorithm is almost similar to the optimal value obtained by the exhaustive search. Second, it is found that the proposed max-min resource allocation scheme is more capable of guaranteeing fairness among BDs, compared with the sum throughput maximization scheme.

The rest of this paper is organized as follows. Section 2 introduces the system model for the general CBN with multi-BD. In section 3, the problem to maximize the minimum throughput of BDs is formulated, which considers the joint optimization of the transmit power of primary user, the backscattering time and reflection coefficients of BDs. In section 4, we analyze the optimization problem and propose an iterative algorithm to solve it for the resource allocation in multi-BD CBN. Section 5 studies the solution of the optimization problem in $\mathrm{CBN}$ with a single BD. In section 6 , the performance of the proposed scheme is evaluated by numerical simulations. Section 7 concludes this paper.

\section{Related work}

System model

Figure 1 system model. 
As depicted in Fig.1, we consider a cognitive backscatter network with a primary transmitter (PT), a cooperative receiver (C-Rx) and $K$ backscatter devices (BDs). The transmission from $\mathrm{PT}$ to $\mathrm{C}$-Rx forms the direct link, and PT to $k$-th $\mathrm{BD}$ then to $\mathrm{C}-\mathrm{Rx}$ forms the backscatter link, for $k=1, \ldots, K$. The PT transmits RF signals to $\mathrm{C}-\mathrm{Rx}$ by active transmissions. Each $\mathrm{BD}$ is equipped with the backscatter module and energy harvesting circuit so that $\mathrm{BD}$ can backscatter information to C-Rx and can harvest energy to power its circuit. C-Rx is designed to recover the signal from the PT as well as the BDs.

The blocking flat fading channel model is considered in this paper. This means that the channel coefficient remains consistent within one time block and may be changed from one to another. Denote $g, h_{1 k}$ and $h_{2 k}$ as the channel coefficient of the direct link, the $\mathrm{PT}$ to $k$-th $\mathrm{BD}$ link and the $k$-th $\mathrm{BD}$ to $\mathrm{C}$-Rx link, respectively. In order to obtain the performance bound, we assume perfect channel state information (CSI) for all links.

An entire time block is divided into $K$ slots, i.e., $\tau_{1}, \tau_{2} \ldots \tau_{K}$, followed by the time division multiple access (TDMA) protocol. PT broadcasts RF signals in an entire block, while the $K$ BDs take turn to operate in the backscatter communication mode. Particularly in $\tau_{K}$, the $k$-th BD utilizes the RF signals to backscatter information to $\mathrm{C}-\mathrm{Rx}$, and the non-backscattering BDs operate in the energy harvesting mode.

Figure 2 Block diagram of transmission link with Hls

Let $s$ denote the transmitted signal by PT and satisfy $\mathbb{E}\left[|s|^{2}\right]=1$, where $\mathbb{E}[$.$] is$ the statistical expectation. As shown in Fig.2, the received signals at the $k$-th $\mathrm{BD}$ in $\tau_{k}$ can be expressed as

$$
y_{S T, k}=h_{1 k} \sqrt{P}\left(s+\eta_{p t}\right)
$$

where $P$ is the transmit power of $\mathrm{PT} ; \eta_{p t} \sim C N\left(0, \kappa_{p t}^{2}\right)$ represents the hardware distortion caused by the PT's RF front ends $[13,17]$, and the parameter $\kappa_{p t}$ reflects the level of HWIs; $\sqrt{P} h_{1 k} \eta_{p t}$ follows a Gaussian distribution with zero mean and variance $\kappa_{p t}^{2} P\left|h_{1 k}\right|^{2}$. Notice that the power of thermal noise is very small and can be ignored at BDs due to only the passive components included and little signal processing operation involved in its circuit $[6,9,18]$.

In $\tau_{k}$, the $k$-th $\mathrm{BD}$ operates in the backscatter communication mode, and splits the received signal into two parts by adjusting the reflection coefficient $\alpha_{k}$ : $\sqrt{1-\alpha_{k}} y_{S T, k}$ for energy harvesting, $\sqrt{\alpha_{k}} y_{S T, k}$ for information transmitting. To better reflect the amount of the harvested energy at the $k$-th $\mathrm{BD}$, the nonlinear $\mathrm{EH}$ model proposed in [19] is considered. Therefore, the total harvested energy of the $k$-th BD can be calculated by

$$
E_{k}^{h}\left(\alpha_{k}, \tau_{k}, P\right)=\Psi\left(P_{k, b}^{i n}\right) \tau_{k}+\Psi\left(P_{k, h}^{i n}\right) \sum_{i=1}^{k-1} \tau_{i} .
$$

In Eq.(2), the first term $\Psi\left(P_{k, b}^{i n}\right) \tau_{k}$ denotes the harvested energy of the $k$-th BD during its backscatter time, where $P_{k, b}^{i n}=P\left(1-\alpha_{k}\right)\left|h_{1 k}\right|^{2}\left(1+\kappa_{p t}^{2}\right)$; The second 
term $\Psi\left(P_{k, h}^{i n}\right) \sum_{i=1}^{k-1} \tau_{i}$ represents the harvested energy of the $k$-th BD during the slots $\tau_{i},(i=1,2, \ldots, k-1)$, where $P_{k, h}^{i n}=P\left|h_{1 k}\right|^{2}\left(1+\kappa_{p t}^{2}\right) ; \Psi(x)=\frac{a x+b}{x+c}-\frac{b}{c}$, is the nonlinear EH mode where $x$ denotes the input power and the correlation coefficients $a, b, c$ can be obtained by fitting curves.

During $\tau_{k}$, the received signal at $\mathrm{C}-\mathrm{Rx}$ is given by

$$
y_{R}=\underbrace{g \sqrt{P}\left(s+\eta_{p t}\right)}_{\text {the first term }}+\underbrace{h_{2 k} \sqrt{\alpha_{k}} y_{S T, k} x_{k}}_{\text {the scond term }}+\eta_{r, k}+n_{r r}
$$

where the first term is the signal of the direct link and the second term is the $k$-th BD's backscatter signal, $n_{r r}$ is the additive white Gaussian noise with zero mean and variance of $\sigma_{2} ; x_{k}$ with unit power is the signal from the $k$-th $\mathrm{BD} ; \eta_{r, k}$ is the distorted noise caused by the C-Rx's RF front ends. Note that $\eta_{r, k}$ consists of two parts: $\eta_{r 1}$ and $\eta_{r 2, k}$, where $\eta_{r 1} \sim C N\left(0, P|g|^{2} \kappa_{r r}^{2}\right)$ and $\eta_{r 2, k} \sim C N\left(0, \alpha_{k} P\left|h_{1 k}\right|^{2}\left|h_{2 k}\right|^{2} \kappa_{r r}^{2}\right)$. Hereby, the parameter $\kappa_{r r}$ reflects the level of HWIs at C-Rx. The multiplicative part $\sqrt{P} g \eta_{p t}$ follow the Gaussian distribution with zero mean and variance $\kappa_{p t}^{2}|g|^{2} P$.

Assume that successive interference cancellation (SIC) technology is employed at $\mathrm{C}-\mathrm{Rx}$ to decode the received signals. The primary signals can be viewed as a fading channel for the backscatter signals which is modulated on the primary signals. In order to achieve coherent detection, C-Rx has to decode the primary signal first[20]. Specifically, the C-Rx firstly decodes the primary signals and then subtracts it to recover the backscatter signals. Thus the signal-interference-noise-ratio (SINR) to decode the primary signal can be obtain from the following Eq.(4) as

$$
\gamma_{k}^{(1)}\left(\alpha_{k}, P\right)=\frac{P|g|^{2}}{P|g|^{2} \kappa_{A}^{2}+\alpha_{k} P\left|h_{1 k}\right|\left|h_{2 k}\right|^{2}\left(1+\kappa_{A}^{2}\right)+\sigma^{2}},
$$

where $\kappa_{A}^{2}=\kappa_{p t}^{2}+\kappa_{r r}^{2}$.

Accordingly, the SINR to decode the $k$-th BD's backscatter signal with imperfect SIC can be written as

$$
\gamma_{k}^{(2)}\left(\alpha_{k}, P\right)=\frac{\alpha_{k} P\left|h_{1}\right|^{2}\left|h_{2}\right|^{2}}{\xi P|g|^{2}\left(1+\kappa_{A}^{2}\right)+\alpha_{k} P\left|h_{1 k}\right|\left|h_{2 k}\right|^{2} \kappa_{A}^{2}+\sigma^{2}},
$$

where $0 \leq \xi \leq 1$ is the interference residual factor which quantifies the level of residual interference. Particularly, $\xi=0$ indicates the perfect SIC and the other values of $\xi$ represent imperfect SIC at the process of recovering backscatter signal[21].

According to the Shannon capacity formula, the achievable throughput of $k$-th $\mathrm{BD}$ can be calculated by

$$
R_{k}\left(\alpha_{k}, \tau_{k}, P\right)=\tau_{k} W \log _{2}\left(1+\Gamma \gamma_{k}^{(2)}\right)
$$

where $\Gamma$ denotes the performance gap caused by the simple modulation of BDs[22]. 


\section{Methods}

Problem formulation

In order to guarantee the communication quality fairness, we maximize the minimum throughput among all BDs via jointly optimizing the PT's transmit power, BDs' backscattering time and reflection coefficients while satisfying the QoS of primary user and the energy causality constraints of BDs. Therefore, the optimization problem can be expressed by

$$
\begin{gathered}
\text { P0: } \max _{P, \alpha_{k}, \tau_{k}} \min _{k} R_{k} \\
\text { s.t.C1 }: 0 \leq \alpha_{k} \leq 1, \forall k \\
\text { C2 }: \sum_{i=1}^{K} \tau_{i} \leq 1, \forall k \\
\text { C } 3: \gamma_{k}^{(1)} \geq \gamma_{\mathrm{th}}, \forall k \\
\text { C4 }: E_{k}^{h} \geq P_{\mathrm{c}} \tau_{k}, \forall k \\
\text { C5 }: 0 \leq P \leq P_{\max },
\end{gathered}
$$

where $P_{c}$ denotes the minimum circuit consumption for all BDs; $\gamma_{\text {th }}$ denotes the minimum threshold for decoding the primary signal.

In P0, C1 and $\mathrm{C} 2$ are the practical constraints of backscattering time and reflection coefficients; C3 ensures the QoS for the communication of primary user; C4 maintains the energy-casuality constraints valid, i.e., the harvested energy is sufficient to cover the power consumption for each BD; C5 considers the transmit power is less than the maximum transmit power $P_{\max }$.

It can be observed that the formulated problem is non-convex and difficult to solve, due to the following two reasons: 1) The objective function is a logarithm function with the fractional form where both numerator and denominator have coupled variables $\left.\alpha_{k} * P ; 2\right)$ The variables $\alpha_{k}, \tau_{k}$ and $P$ are coupled in C3 and C4. Thus the traditional convex optimization methods cannot be applied directly to solve this problem.

\section{A Resource allocation in multi-BD CBN}

In order to solve P0, we adopt the following steps. First, we introduce a slack variable to transform the complex objective function into a affine function. And next, we determine the optimal value of $P$ and decouple it from other optimization variables. Finally, we propose an iterative algorithm to solve the transformed problem based on the block coordinate descent technique.

By introducing the slack variable $Q$, the problem $\mathbf{P 0}$ is converted to

$$
\begin{aligned}
& \text { P1: } \max _{Q, P, \alpha_{k}, \tau_{k}} Q \\
& \text { s.t. } \mathrm{C} 1 \sim \mathrm{C} 5, \\
& \quad \mathrm{C} 6: R_{k}^{\min } \geq Q, \forall k,
\end{aligned}
$$

where $R_{k}^{\min }$ in $\mathrm{C} 6$ denotes the minimum throughput of the multi-BD. In addition, C6 ensures the QoS of communication for each BD.

Theorem1. The optimal transmit Power is $P_{\max }$, i.e., $P^{*}=P_{\max }$, where "* * represents the optimal solution. 
Proof. Dividing both numerator and denominator by $P$, one we can see that the Eqs.(3), (4) and (5) are increasing functions with respect to the optimization variable $P$. One can also see from Eq.(2), the harvested energy of the BDs increases with $P$. Based on the above description, we conclude that the probability to satisfy constraints $\mathrm{C} 3-\mathrm{C} 6$ increases with $P$. Combining the above conclusion with the $\mathrm{C} 5$, it is not hard to know $P^{*}=P_{\max }$, where ${ }^{*}$ denotes the optimal value. Theorem1 is proved.

Substituting $P_{\max }$ into the problem $\mathbf{P} \mathbf{1}$, we can simplify $\mathbf{P} \mathbf{2}$ as

$$
\begin{aligned}
& \text { P2 } \max _{Q, \alpha_{k}, \tau_{k}} Q \\
& \text { s.t.C1 } 0 \leq \alpha_{k} \leq 1, \forall k \\
& \quad \text { C2 }: \sum_{i=1}^{K} \tau_{i} \leq 1, \forall k \\
& \text { C3 }-1: \gamma_{k}^{(1)}\left(\alpha_{k}, \tau_{k}, P_{\max }\right) \geq \gamma_{\mathrm{th}}, \forall k \\
& \text { C4 }-1: E_{k}^{h}\left(\alpha_{k}, \tau_{k}, P_{\max }\right) \geq P_{\mathrm{c}} \tau_{k}, \forall k \\
& \text { C6 }-1: R_{k}^{\min }\left(\alpha_{k}, \tau_{k}, P_{\max }\right) \geq Q, \forall k .
\end{aligned}
$$

Despite the problem $\mathbf{P 2}$ reduces one optimization variable compared with $\mathbf{P 1}$, it is still non-convex because of the coupled optimization variables $\alpha_{k}$ and $\tau_{k}$. To cope with this, we propose a BCD-based iterative algorithm [23]. In particular, the problem $\mathbf{P 2}$ is decoupled into two convex subproblems, i.e., optimizing the backscattering time $\tau_{k}$ for a given reflection coefficient $\alpha_{k}$ and optimizing the $\alpha_{k}$ for a fixed $\tau_{k}$.

First, for a given reflection coefficient $\alpha_{k}^{\{l\}}$, we can obtain the backscattering time by solving the following linear programming problem in $l$-th iteration, given as

$$
\begin{aligned}
& \text { P3 }: \max _{Q, \tau_{k}} Q \\
& \text { C2 }: \sum_{i=1}^{K} \tau_{i} \leq 1, \forall k \\
& \text { C3 }-2: \gamma_{k}^{(1)}\left(\alpha_{k}^{\{l\}}, P_{\max }\right) \geq \gamma_{\mathrm{th}}, \forall k \\
& \mathrm{C} 4-2: E_{k}^{h}\left(\alpha_{k}^{\{l\}}, \tau_{k}, P_{\max }\right) \geq P_{\mathrm{c}} \tau_{k}, \forall k \\
& \mathrm{C} 6-2: R_{k}^{\min }\left(\alpha_{k}^{\{l\}}, \tau_{k}, P_{\max }\right) \geq Q, \forall k .
\end{aligned}
$$

Second, for a given backscattering time $\tau_{k}^{\{l\}}$, the reflection coefficient can be obtained by solving the following problem in $l$-th iteration, i.e.,

$$
\begin{aligned}
& \text { P4 } \max _{Q, \alpha_{k}} Q \\
& \text { s.t.C } 1: 0 \leq \alpha_{k} \leq 1, \forall k \\
& \quad \text { C } 3-3: \gamma_{k}^{(1)}\left(\alpha_{k}, P_{\max }\right) \geq \gamma_{\mathrm{th}}, \forall k \\
& \quad \text { C4 }-3: E_{k}^{h}\left(\tau_{k}^{\{l\}}, \alpha_{k}, P_{\max }\right) \geq P_{\mathrm{c}} \tau_{k}, \forall k \\
& \quad \text { C6 }-3: R_{k}^{\min }\left(\tau_{k}^{\{l\}}, \alpha_{k}, P_{\max }\right) \geq Q, \forall k .
\end{aligned}
$$

$\mathbf{P} 4$ is a convex problem and the detailed proof can be found in Appendix A. Therefore, $\mathbf{P} 4$ can be efficiently solved by CVX [24]. 
Based on the above analysis, we propose a BCD-based iterative algorithm to solve P2, as summarized in Algorithm 1.

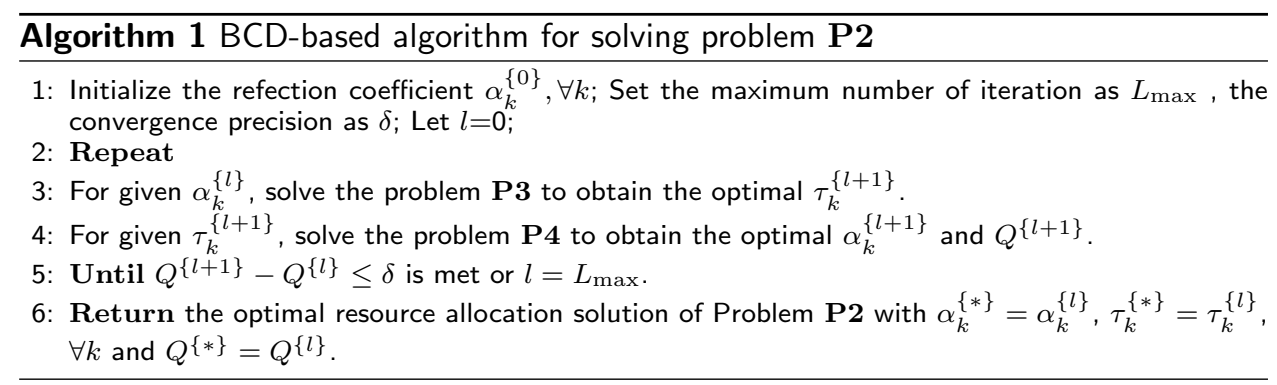

The convergence of Algorithm 1 is guaranteed because the convex problems P3 and P4 in each iteration can be solved by CVX [25]. Assume that the interior point method is adopting to solve the two subproblems and the number of convergence is $N_{c}$. The computational complexity of $\mathbf{P 3}$ and $\mathbf{P 4}$ are $O(\sqrt{(m)} \log (m)), O(\sqrt{(n)} \log (n))$ where $m$ and $n$ represent the number of constraints respectively[26, 27]. Therefore, the computational complexity of Algorithm 1 is polynomial, i.e., $N_{c} O(\sqrt{m n} \log (m) \log (n))$ by multiplying the computational complexity solving the convex problems P3 and P4.

B Resource allocation in single-BD CBN

To obtain the valuable analytical results, we consider the single-BD CBN, i.e., $K=1$, and discuss the resource allocation scheme in this section. For the single-BD scenario, there is no need to consider the backscattering time allocation. Therefore, the resource allocation problem for the single-BD CBN can be written as

$$
\begin{aligned}
& \text { P5 }: \max _{P, \alpha} R \\
& \text { s.t.C1 }{ }^{\prime}: 0 \leq \alpha \leq 1 \\
& \mathrm{C} 2^{\prime}: \gamma^{(1)} \geq \gamma_{\mathrm{th}} \\
& \mathrm{C} 3^{\prime}: E(\alpha, P) \geq P_{c} \\
& \mathrm{C} 4^{\prime}: 0 \leq P \leq P_{\max } .
\end{aligned}
$$

P5 is non-convex due to the existing of coupled variables $\alpha$ and $P$ in the objective function and the constraint $\mathrm{C}^{\prime}$. Nevertheless, one observation from the objective function is that, the left-hand side of constraints $\mathrm{C} 2^{\prime}$ and $\mathrm{C} 3^{\prime}$ all increase with $P$. Taking $\mathrm{C}_{5}^{\prime}$ into account, the optimal transmit power can be determined as $P_{\max }$, which is consistent with Theorem 1 . Besides, the optimal value of $\alpha$ can be obtained by Lemma1.

Lemma1. The maximum feasible $\alpha$ is

$\alpha_{m}=\left\{\min \left[1-\frac{c P_{c}}{P_{\max }\left|h_{1}\right|^{2}\left(1+\kappa_{\mathrm{A}}^{2}\right)\left(a c-b-c P_{c}\right)}, \frac{|g|^{2}-\gamma_{\mathrm{th}}\left(|g|^{2} \kappa_{\mathrm{A}}^{2}+\sigma_{0}^{2}\right)}{\gamma_{\mathrm{th}}\left|h_{1}\right|^{2}\left|h_{2}\right|^{2}\left(1+\kappa_{\mathrm{A}}^{2}\right)}\right]\right\}^{+}$

where $\sigma_{0}^{2}=\frac{\sigma^{2}}{P_{\max }}$ and $\{x\}^{+}$represents $\max \{0, x\}$. 
Proof. Given the optimal value of transmit power $P_{\max }$, we can obtain the low bound of $\alpha$ in constraint $\mathrm{C} 2$, which can be derived by

$$
\alpha_{1} \leq \frac{P_{\max }|g|^{2}-\gamma_{\mathrm{th}}\left(P_{\max }|g|^{2} \kappa_{\mathrm{A}}^{2}+\sigma^{2}\right)}{\gamma_{\mathrm{th}} P_{\max }\left|h_{1}\right|^{2}\left|h_{2}\right|^{2}\left(1+\kappa_{\mathrm{A}}^{2}\right)} .
$$

Similarly, the low bound value of $\alpha$ in constraint $\mathrm{C} 3^{\prime}$ can be obtained by

$$
\alpha_{2} \leq 1-\frac{c P_{c}}{P_{\max }\left|h_{1}\right|^{2}\left(1+\kappa_{\mathrm{A}}^{2}\right)\left(a c-b-c P_{c}\right)} .
$$

Combining the above analysis with the constraint $\mathrm{C1}^{\prime}$, the largest $\alpha$ in feasible region is given by $\max \left\{0, \min \left\{\alpha_{1}, \alpha_{2}\right\}\right\}$. Lemma1 is proved.

Theorem 2. The optimal solution of $\mathbf{P 5}$ is given by

$$
\left\{\begin{array}{l}
\alpha^{*}=\alpha_{m} \\
P^{*}=P_{\max }
\end{array}\right.
$$

Proof. In P5, we can observe that the objective function monotonically increases with $P$ for any given feasible $\alpha$. In order to maximize the throughput, the optimal $P$ should choose the maximum value $P_{\max }$, i.e., $P^{*}=P_{\max }$. It also can be seen from $\mathbf{P 5}$ that the throughput expression is also a growth function with $\alpha$ for any given feasible $P$. Thus the maximum throughput is obtained at the largest value of reflection coefficient, i.e., $\alpha_{m}$, which is verifiably feasible when $P^{*}=P_{\max }$ in Lemma1. Based on the above analysis, the values of optimization variables are same as Theorem 2 .

Theorem 2 provides an analytical solution for P5 and reveals some insights for the considered single-BD scenario. For example, PT adopts the maximum power $P_{\max }$ can improve the throughput performance of BD. And for the energy causality constraint and the primary user's QoS constraint, higher the $P$, higher is the probability to satisfy the constraints.

\section{Results and discussion}

In this section, we conduct several simulation experiments to analyze the performance of BDs in CBN. Assume that all the involved channels feature Rayleigh fading with unit variance as small-scale fading and distance exponential fading as large-scale fading. Thus the channel gains can be modeled by $|g|^{2} d_{0}^{-3},\left|h_{1 k}\right|^{2} d_{1 k}^{-3}$ and $\left|h_{2 k}\right|^{2} d_{2 k}^{-3}$, where $d_{0}$ and $d_{1 k}$ are the distance from PT to IR and $k$-th BD, and $d_{2 k}$ is the distance from $k$-th $\mathrm{BD}$ to IR respectively. We set $d_{0}=7 \mathrm{~m}, d_{11}=2.5 \mathrm{~m}$, $d_{12}=4 \mathrm{~m}$ and $d_{21}=3.5 \mathrm{~m}, d_{22}=3 \mathrm{~m}$, respectively. For the nonlinear EH model, we set the correlation parameters $a=2.463, b=1.735, c=0.826$. In addition, we also assume the same level of HWIs exists at the RF front ends as $\kappa=\kappa_{p t}=\kappa_{r r}=0.1$. The other parameter settings are as shown in Table 1.

Figure 3 Convergence performance. 
Table 1 Simulation parameter settings

\begin{tabular}{ccc}
\hline Parameters & Symbols & value \\
\hline Bandwidth & $W$ & $10 \mathrm{MHz}$ \\
Noise power & $\sigma_{2}$ & $-114 \mathrm{dBm}$ \\
SINR threshold & $\gamma_{\text {th }}$ & $3 \mathrm{~dB}$ \\
path-loss exponent & $v$ & 3 \\
Performance gap & $\Gamma$ & 0.1 \\
Interference residual factor & $\xi$ & 0.01 \\
\hline
\end{tabular}

Figure $4 \mathrm{Max}-\mathrm{min}$ throughput (bits/Hz) versus the optimal transmit power $P_{\max }$ with different decoding threshold $\gamma_{\text {th }}$ of primary user.

Figure 3 depicts the convergence performance of the proposed resource allocation scheme, i.e., Algorithm 1. As shown in this figure, the max-min throughout by proposed algorithm converges within 10 iterations. Also, it can be seen that the convergence value of the max-min throughput by Algorithm 1 is quite close to the optimal result by exhaustive search method, which indicates the well convergence performance of the proposed iterative algorithm.

Figure $5 \mathrm{Max}-\mathrm{min}$ throughput (bits $/ \mathrm{Hz}$ ) versus the minimum power consumption with different interference residual factor $\xi$ for $P_{\max }=5 \mathrm{~dB}$.

Figure $6 \mathrm{Max}-\mathrm{min}$ throughput (bits/Hz) versus the distance of PT-BD with different level of HIs for $P_{\max }=5 \mathrm{dBm}$.

The max-min throughput based on the proposed resource allocation scheme versus the optimal transmit power is depicted in Figure 4. As in the theoretical analysis, the max-min throughput increases with the optimal transmit power $P_{\max }$. Additionally, the impact of SINR threshold on the max-min throughput also can be seen in this figure, where the max-min throughput trends down with increasing SINR threshold of the primary user. The reason for this is that lower SINR threshold reflects higher interference tolerance. In order to cater for the QoS of primary user, BDs have to reduce the transmit power with increased SINR threshold.

Figure 5 shows the max-min throughput performance versus the minimum power consumption of BDs under different conditions of interference residual factor. The performance curve tends to decline as the minimum power consumption increase. The internal reasons can be drawn that BDs cannot but reducing the reflection coefficient to harvest more energy to meet the energy casuality constraint, however, the throughput function monotonically increases with respect to the reflection coefficient as in Eq.(7). Moreover, we can also see that as the interference residual factor increases, the max-min throughput decreases for any given $P_{c}$. This is due to that, the interference residual factor quantifies the unremoved interference of primary signals which degrades the performance of BDs.

Figure 6 plots the max-min throughput versus the distance from PT to BD in CBN with a single BD. It can be observed that the increase in distance causes a downward trend in max-min throughput. This is because the channel gain is 
inversely proportional to distance. BD needs to reduce the reflection coefficient for harvesting sufficient energy to sustain its own circuit with the distance growth, which leads decaying throughput. Besides, we can observe the max-min throughput degrades before the intersection of the curves as $\kappa$ increases from 0.2 to 0.3 , but the contrary is shown after the intersection. This is due to the fact that the HWIs can compensate part of the harvested energy as described by Eq.(2), which improves the performance in higher distance regime.

Figure 7 Comparison of throughput under max-min scheme and sum throughput maximization scheme.

Take the sum throughput maximization resource allocation scheme as benchmark scheme for comparison. It is important to note that Algorithm 1 also works for the benchmark scheme to obtain the resource allocation management. Figure 7 shows the comparison of the proposed scheme based on max-min criterion with the benchmark scheme. First, we can visualize that the difference of throughput between the best and worst links from the proposed max-min scheme is less than the benchmark scheme whether in the scenarios of two or four BDs. Second, the difference of throughput between the best and worst links from the benchmark has increased nearly threefold as the number of users increases from 2 to 4 , which is significantly larger than the difference increase of the proposed max-min scheme. Third, the average throughput of benchmark scheme is slightly outperform than the proposed max-min scheme. This is because, benchmark scheme inclines to the users with good channel status to obtain the maximum of sum throughput, while the proposed scheme maximizes the throughput of user with worst link status to guarantee much fairness of inter-BD.

\section{Conclusion}

In this paper, we studied the max-min resource allocation scheme considering the nonlinear EH model and the existence of HWIs. Specifically, we formulate the joint PT's transmit power, BDs' backscattering time and reflection coefficients optimization problem for maximizing the minimum throughput of BDs, and propose an iterative algorithm based on BCD to solve the optimization problem. In addition, we obtained the closed-form solution of the optimization problem in the special scenario for the CBN with single-BD. Simulation results demonstrated the proposed max-min resource allocation scheme efficiently ensures fairness among BDs and revealed the effect of different relevant parameters like the QoS of primary user, distance from PT to BD, the level of HWIs, etc.

\section{Appendix A}

In P4, the constraint C3-3 can be transformed to a linear constraint as described in Eq.(14). The objective function and constraint $\mathrm{C} 1$ are affine. Therefore, we just need to discuss the concavity of the constraints C4-3 and C6-3.

For the constraint C4-3, the second term of the left-hand side is a constant independent of the variables, thus we only discuss the first term marked by $E_{k}(x)$. 
Taking the second order derivative of $E_{k}(x)$ yields

$$
\frac{\partial^{2} E_{k}}{\partial x^{2}}=\frac{(b-a c)\left(\left|h_{1 k}\right|^{2}\left(1+\kappa_{B}^{2}\right) P_{\max }\right)^{2}}{\left(\left(1-\alpha_{k}\right)\left|h_{1 k}\right|^{2}\left(1+\kappa_{B}^{2}\right) P_{\max }+c\right)^{3}},
$$

where the positivity and negativity is determined by $b-a c$ and $c$. According to the non-negativity and saturation properties of nonlinear EH model, the correlations between the parameters in the adopted model are proved in [28] as $b-a c<0$ and $c>0$. Thus $\frac{\partial^{2} E_{k}}{\partial x^{2}}<0$ and C4-3 is a convex constraint.

For the constraint $\mathrm{C} 6-3$, it has the same structure as $H(x)=\log \left(1+\frac{A x}{B x+C}\right)$ where the parameters $\mathrm{A}, \mathrm{B}$ and $\mathrm{C}$ are all above zero. Taking the second order derivative of $H(x)$, we can derive

$$
\frac{\partial^{2} H}{\partial x^{2}}=-\frac{\left(2 A B x+2 B^{2} x+2 B C+A C\right)^{2} C}{[(A x+B x+C)(B x+C)]^{2} \ln 2} .
$$

In Eq.(18), we can visualize that $\frac{\partial^{2} H}{\partial x^{2}}<0$. Thus the left-hand side of C6-3 is a concave function. This means C6-3 is a convex constraint.

From the analyses made above, we can draw the conclusion that $\mathbf{P} \mathbf{4}$ is a convex problem[29].

\section{Abbreviations}

CBN: cognitive backscatter networks; BD: Backscatter devices; C-Rx: Cooperative receiver; HWIs: hardware impairments; EH: Energy harvesting; IoT: Internet-ofthings; QoS: Quality of service; CR: Cognitive radio; ADC: Analog-digital converters; RF: Radio frequency; PT: Primary transmitter; AmBC: Ambient backscatter communication; HPA: High-power-amplifier; TDD: Time-division-multiplexing; CSI: Channel state information; SIC: Successive interference cancellation; BCD: Block coordinate descent.

\section{Declarations}

Availability of data and materials

Data sharing not applicable to this article as no datasets were generated or analysed during the current study.

Competing interests

The authors declare that they have no competing interests.

Funding

This work was supported by the Science and Technology Innovation Team of Shaanxi Province for Broadband Wireless and Application under Grant 2017KCT-30-02 and the Natural Science Foundation of Shaanxi Province under Grant 2021JQ-713.

Authors' contributions

SLQ conceived of the study, designed and modeled. GXN carried out experiments, participated in the performance analysis and drafted the manuscript. LGY participated in the design and helped to draft the manuscript. All authors read and approved the final manuscript.

Acknowledgements

No applicable. 


\section{Authors' information}

Xiaona Gao received the B.E. degree from Xuchang University, Henan, China, in 2020. She is currently pursuing the the M.S. degree in the Xi'an University of Posts and Telecommunications, Xi'an, China. Her current research interests include wireless communication technology, and ambient backscatter systems.

Liqin Shi received the B.S. degree from Sichuan University, Chengdu, China, in 2015, and the Ph.D. degree from Xidian University, Xi'an, China, in 2020. She was a Joint Ph.D. student with the Department of Electrical and Computer Engineering, Utah State University, Logan, UT, USA, from 2018 to 2019. Since 2020, she has been with the Xi'an University of Posts and Telecommunications, Xi'an, where she is currently an Associate Professor with the Department of Communication and Information Engineering. She has published more than 20 papers in IEEE TRANSACTIONS ON VEHICULAR TECHNOLOGY and IEEE ICC. Her research interests include wireless energy harvesting and mobile-edge computing. Dr. Shi is also a reviewer of multiple international journals, including the IEEE JOURNAL ON SELECTED AREAS IN COMMUNICATIONS and the IEEE TRANSACTIONS ON WIRELESS COMMUNICATIONS. She has served as a TPC member of IEEE SmartloT in 2018 and IEEE VTC-FALL in 2019.

Guangyue Lu received the Ph.D. degree from Xidian University, Xi'an, China, in 1999. From September 2004 to August 2006, he was a Guest Researcher with the Signal and Systems Group, Uppsala University, Uppsala, Sweden. Since 2005, he has been a Full Professor with the School of Communications and Information Engineering, Xi'an University of Posts and Telecommunications, Xi'an. He has been the funded by over twenty projects including the National Natural Science Foundation of China, the 863 Program, and Important National Science and Technology Specific Projects. Due to his excellent contributions in education and research, he was awarded by the Program for New Century Excellent Talents in University, Ministry of Education, China, in 2009. He is currently the Vice President of Xi'an University of Posts and Telecommunications, and the Director of the Shaanxi Key Laboratory of Information Communication Network and Security. His research interests include wireless communications, energy harvesting, cognitive radio and cooperative spectrum sensing.

\section{Author details}

Shaanxi Key Laboratory of Information Communication Network and Security, Xi'an University of Posts \& Telecommunications, Xi'an 710121, China.

\section{References}

1. Rezaei, F., Tellambura, C., Herath, S.: Large-scale wireless-powered networks with backscatter communications-a comprehensive survey. IEEE Open Journal of the Communications Society 1, 1100-1130 (2020). doi:10.1109/OJCOMS.2020.3012466

2. Khan, A.A., Rehmani, M.H., Rachedi, A.: Cognitive-radio-based internet of things: Applications, architectures, spectrum related functionalities, and future research directions. IEEE Wireless Communications 24(3), 17-25 (2017). doi:10.1109/MWC.2017.1600404

3. Ye, Y., Shi, L., Chu, X., Lu, G.: On the outage performance of ambient backscatter communications. IEEE Internet of Things Journal 7(8), 7265-7278 (2020). doi:10.1109/JIOT.2020.2984449

4. Van Huynh, N., Hoang, D.T., Lu, X., Niyato, D., Wang, P., Kim, D.I.: Ambient backscatter communications: A contemporary survey. IEEE Communications Surveys Tutorials 20(4), 2889-2922 (2018). doi:10.1109/COMST.2018.2841964

5. Long, R., Liang, Y.-C., Guo, H., Yang, G., Zhang, R.: Symbiotic radio: A new communication paradigm for passive internet of things. IEEE Internet of Things Journal 7(2), 1350-1363 (2020). doi:10.1109/JIOT.2019.2954678

6. Kang, X., Liang, Y.-C., Yang, J.: Riding on the primary: A new spectrum sharing paradigm for wireless-powered iot devices. IEEE Transactions on Wireless Communications 17(9), 6335-6347 (2018). doi:10.1109/TWC.2018.2859389

7. Wang, J., Ye, H.-T., Kang, X., Sun, S., Liang, Y.-C.: Cognitive backscatter noma networks with multi-slot energy causality. IEEE Communications Letters 24(12), 2854-2858 (2020). doi:10.1109/LCOMM.2020.3019203

8. Zhuang, Y., Li, X., Ji, H., Zhang, H., Leung, V.C.M.: Optimal resource allocation for rf-powered underlay cognitive radio networks with ambient backscatter communication. IEEE Transactions on Vehicular Technology 69(12), 15216-15228 (2020). doi:10.1109/TVT.2020.3037152

9. Xiao, S., Guo, H., Liang, Y.-C.: Resource allocation for full-duplex-enabled cognitive backscatter networks. IEEE Transactions on Wireless Communications 18(6), 3222-3235 (2019). doi:10.1109/TWC.2019.2912203

10. Guo, H., Long, R., Liang, Y.-C.: Cognitive backscatter network: A spectrum sharing paradigm for passive iot. IEEE Wireless Communications Letters 8(5), 1423-1426 (2019). doi:10.1109/LWC.2019.2919835

11. Lyu, B., You, C., Yang, Z., Gui, G.: The optimal control policy for rf-powered backscatter communication networks. IEEE Transactions on Vehicular Technology 67(3), 2804-2808 (2018). doi:10.1109/TVT.2017.2768667

12. Yang, G., Yuan, D., Liang, Y.-C., Zhang, R., Leung, V.C.M.: Optimal resource allocation in full-duplex ambient backscatter communication networks for wireless-powered iot. IEEE Internet of Things Journal 6(2), 2612-2625 (2019). doi:10.1109/JIOT.2018.2872515

13. Liu, Z., Lu, G., Ye, Y., Chu, X.: System outage probability of ps-swipt enabled two-way af relaying with hardware impairments. IEEE Transactions on Vehicular Technology 69(11), 13532-13545 (2020). doi:10.1109/TVT.2020.3030134

14. Costa, E., Midrio, M., Pupolin, S.: Impact of amplifier nonlinearities on ofdm transmission system performance. IEEE Communications Letters 3(2), 37-39 (1999). doi:10.1109/4234.749355

15. Valenta, C.R., Durgin, G.D.: Harvesting wireless power: Survey of energy-harvester conversion efficiency in far-field, wireless power transfer systems. IEEE Microwave Magazine 15(4), 108-120 (2014). doi:10.1109/MMM.2014.2309499 
16. Xu, X., Özçelikkale, A., McKelvey, T., Viberg, M.: Simultaneous information and power transfer under a non-linear rf energy harvesting model. In: 2017 IEEE International Conference on Communications Workshops (ICC Workshops), pp. 179-184 (2017). doi:10.1109/ICCW.2017.7962654

17. Solanki, S., Singh, V., Upadhyay, P.K.: Rf energy harvesting in hybrid two-way relaying systems with hardware impairments. IEEE Transactions on Vehicular Technology 68(12), 11792-11805 (2019). doi:10.1109/TVT.2019.2944248

18. Qian, J., Gao, F., Wang, G., Jin, S., Zhu, H.: Noncoherent detections for ambient backscatter system. IEEE Transactions on Wireless Communications 16(3), 1412-1422 (2017). doi:10.1109/TWC.2016.2635654

19. Chen, Y., Zhao, N., Alouini, M.-S.: Wireless energy harvesting using signals from multiple fading channels IEEE Transactions on Communications 65(11), 5027-5039 (2017). doi:10.1109/TCOMM.2017.2734665

20. Wang, G., Gao, F., Fan, R., Tellambura, C.: Ambient backscatter communication systems: Detection and performance analysis. IEEE Transactions on Communications 64(11), 4836-4846 (2016). doi:10.1109/TCOMM.2016.2602341

21. Luo, L., Li, Q., Cheng, J.: Performance analysis of overlay cognitive noma systems with imperfect successive interference cancellation. IEEE Transactions on Communications 68(8), 4709-4722 (2020) doi:10.1109/TCOMM.2020.2992471

22. Kim, S.H., Kim, D.I.: Hybrid backscatter communication for wireless-powered heterogeneous networks. IEEE Transactions on Wireless Communications 16(10), 6557-6570 (2017). doi:10.1109/TWC.2017.2725829

23. Razaviyayn, M., Hong, M., Luo, Z.Q., Pang, J.S.: Parallel successive convex approximation for nonsmooth nonconvex optimization. advances in neural information processing systems (2014)

24. Grant, M., Boyd, S.: CVX: Matlab Software for Disciplined Convex Programming, version 2.1. http://cvxr.com/cvx (2014)

25. Razaviyayn, M., Hong, M., Luo, Z.Q.: A unified convergence analysis of block successive minimization methods for nonsmooth optimization. Siam Journal on Optimization 23(2), 1126-1153 (2012)

26. Shi, L., Ye, Y., Hu, R.Q., Zhang, H.: Energy efficiency maximization for swipt enabled two-way df relaying. IEEE Signal Processing Letters 26(5), 755-759 (2019). doi:10.1109/LSP.2019.2906463

27. Shi, L., Hu, R.Q., Gunther, J., Ye, Y., Zhang, H.: Energy efficiency for rf-powered backscatter networks using htt protocol. IEEE Transactions on Vehicular Technology 69(11), 13932-13936 (2020) doi:10.1109/TVT.2020.3014500

28. Ye, Y., Shi, L., Chu, X., Lu, G.: Throughput fairness guarantee in wireless powered backscatter communications with htt. IEEE Wireless Communications Letters 10(3), 449-453 (2021). doi:10.1109/LWC.2020.3014740

29. Boyd, S., Boyd, S.P., Vandenberghe, L.: Convex Optimization. Cambridge university press, Cambridge,U.K. (2004) 
Figures

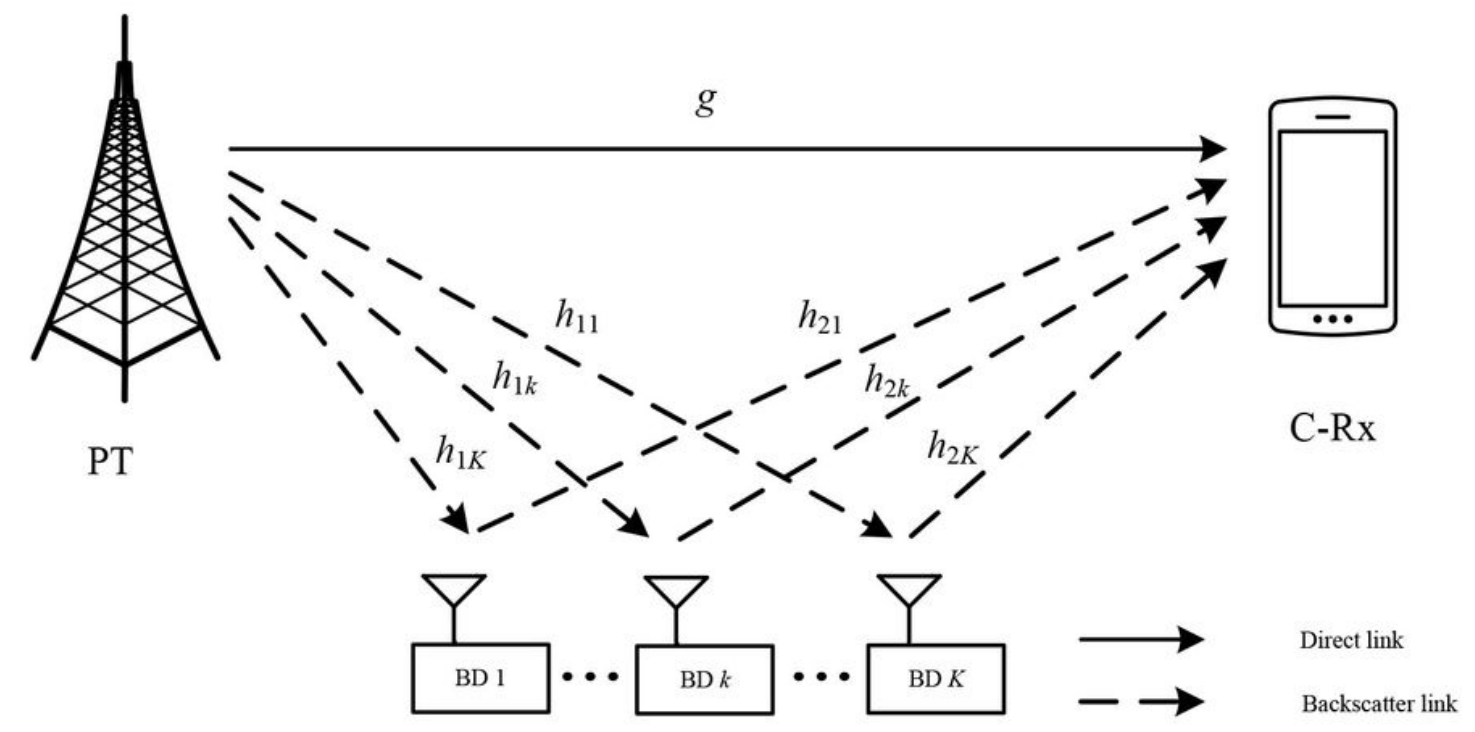

Figure 1

system model.

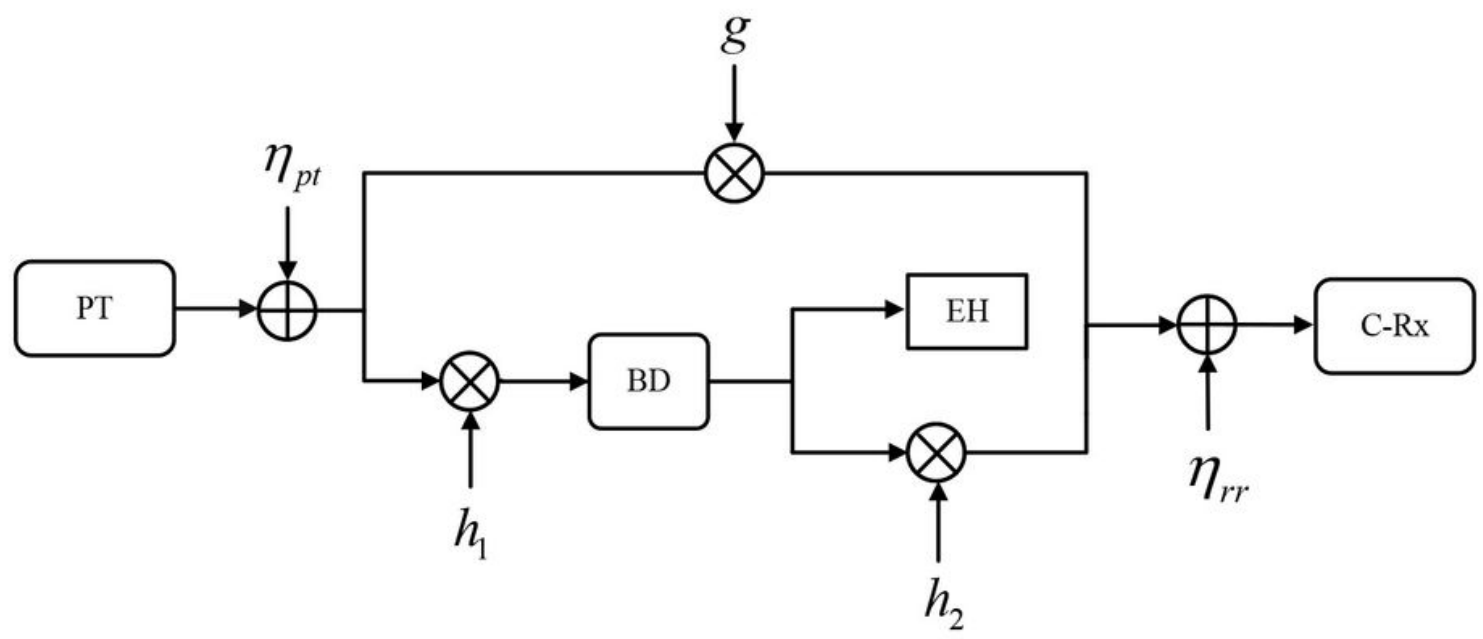


Figure 2

Block diagram of transmission link with HIs

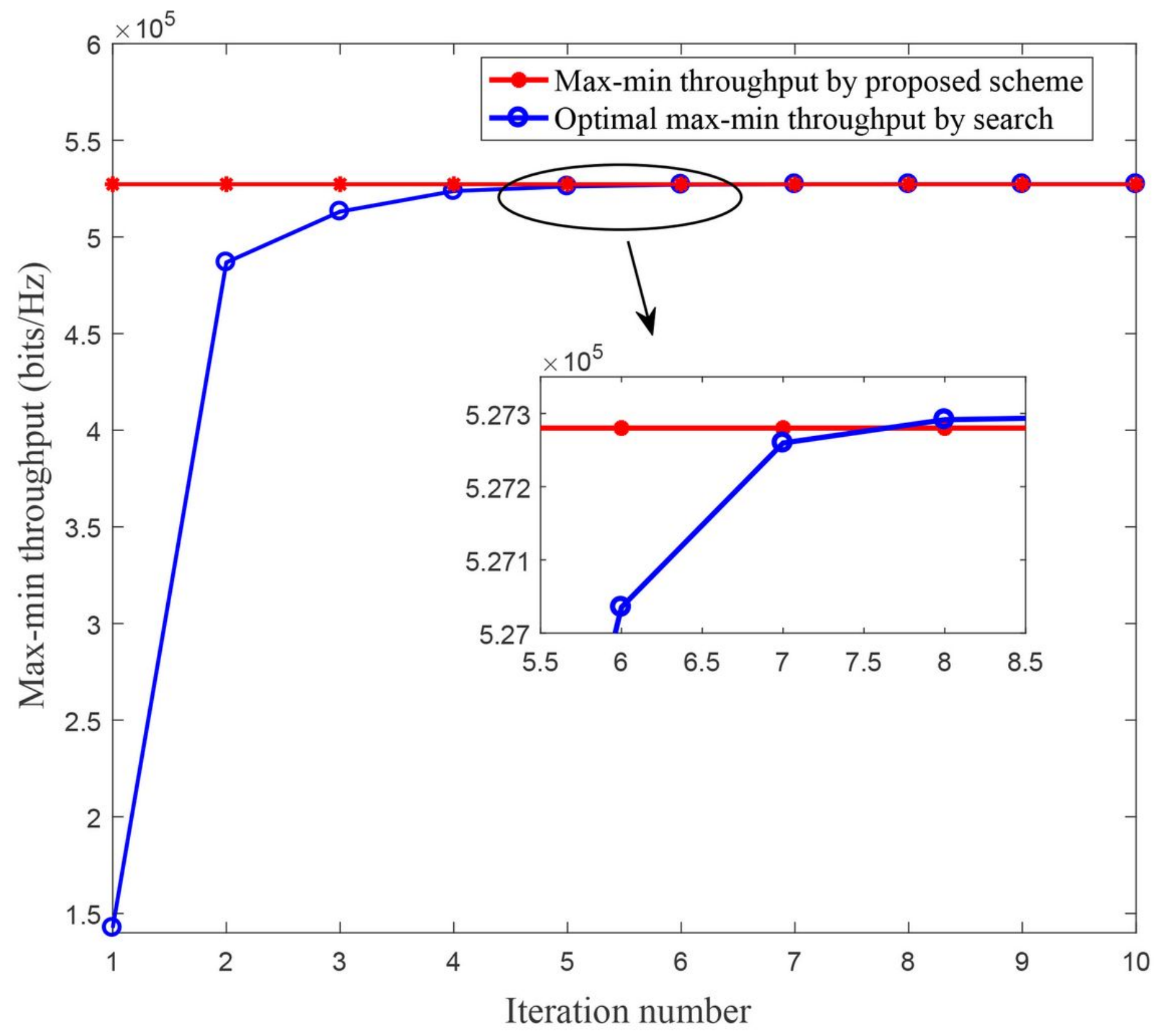

Figure 3

Convergence performance. 


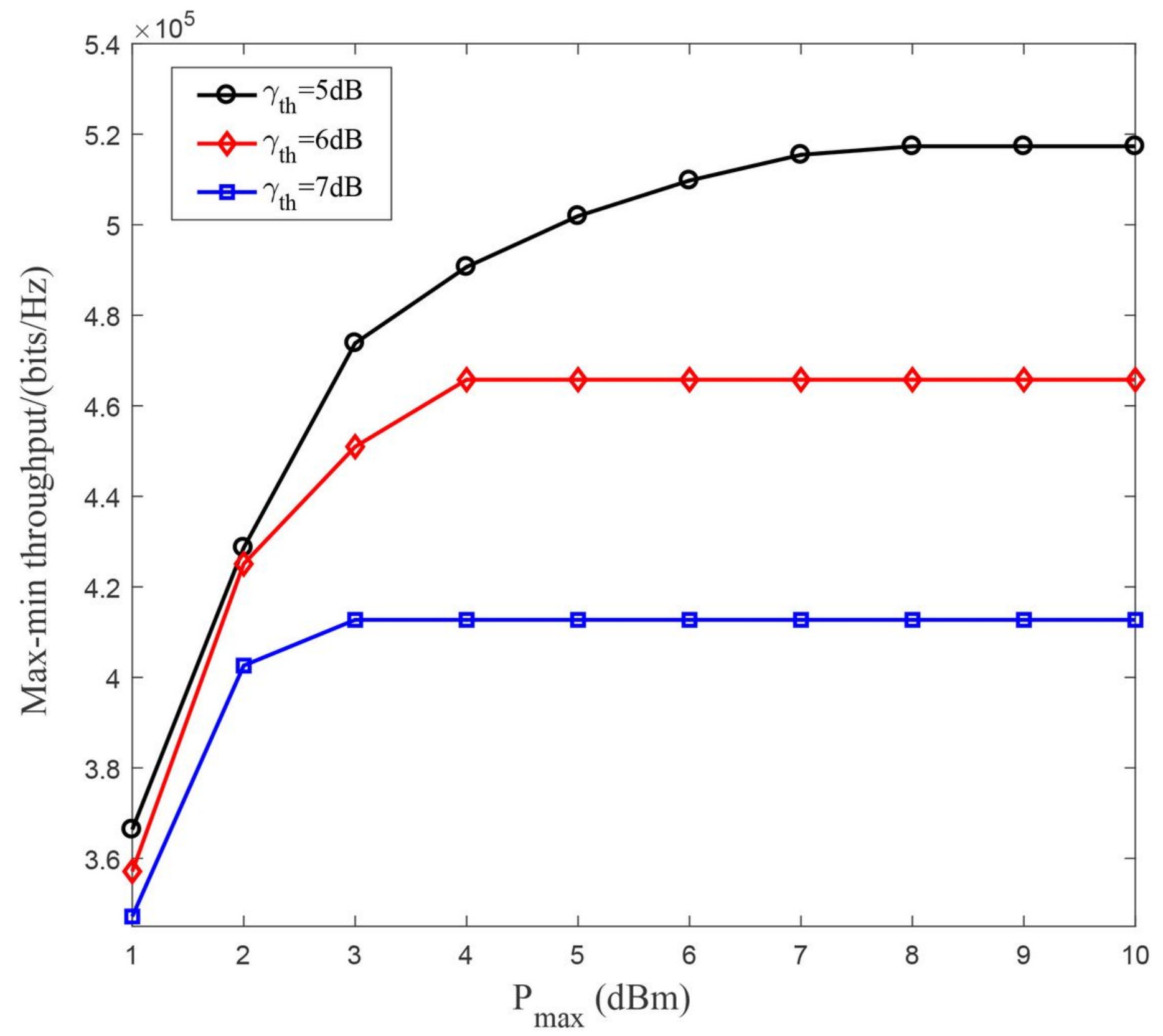

Figure 4

Max-min throughput (bits/Hz) versus the optimal transmit power Pmax with different decoding threshold yth of primary user. 


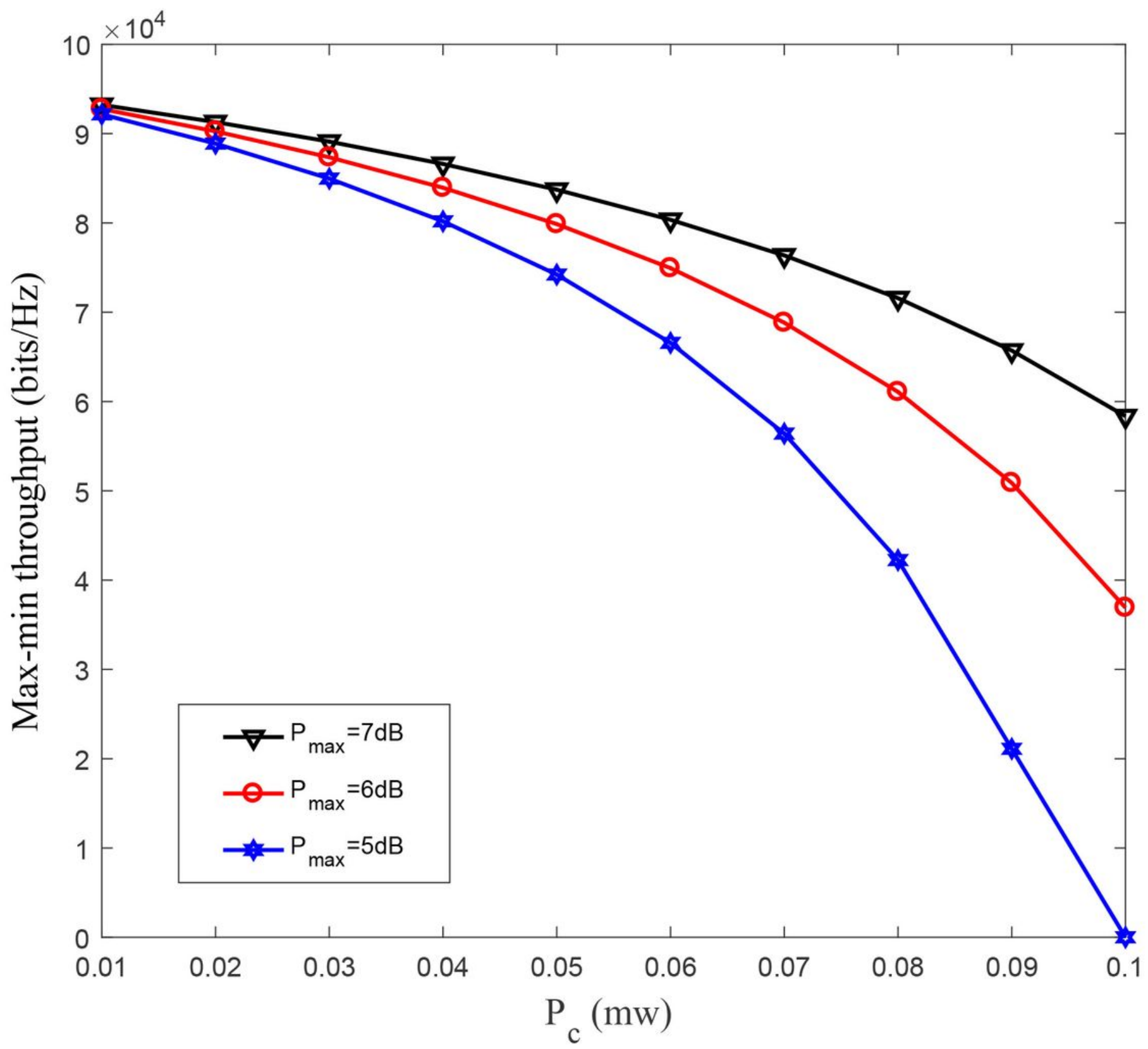

Figure 5

Max-min throughput (bits/ $\mathrm{Hz}$ ) versus the minimum power consumption with different interference residual factor $\xi$ for $P \max =5 \mathrm{~dB}$. 


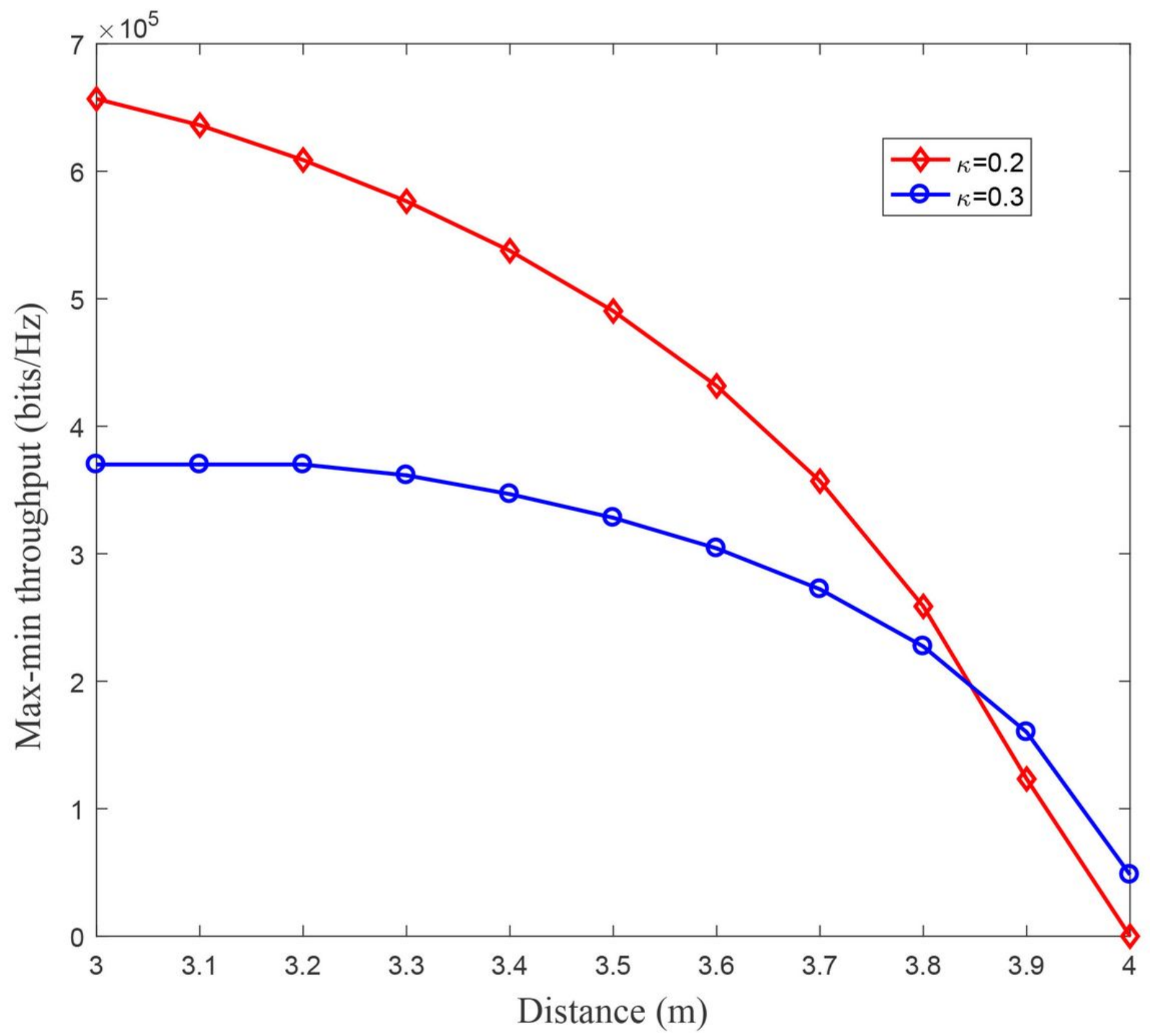

Figure 6

Max-min throughput (bits $/ \mathrm{Hz}$ ) versus the distance of PT-BD with different level of Hls for Pmax $=5 \mathrm{dBm}$. 


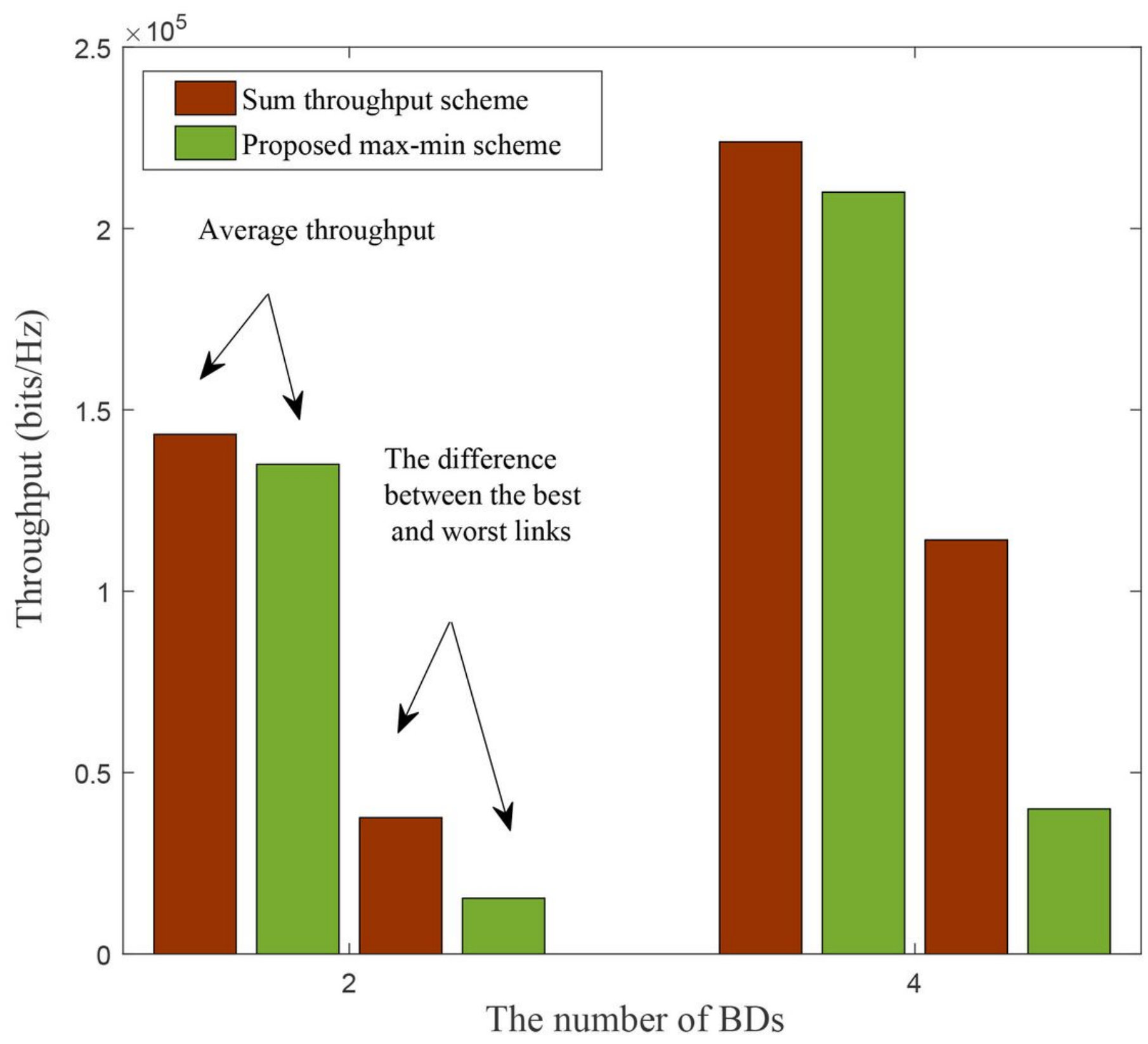

Figure 7

Comparison of throughput under max-min scheme and sum throughput maximization scheme. 